\title{
Method for Testing Sustained Attention in Touchscreen Operant Chambers in Rats
}

Brittany Wicks, David E. Waxler, Kyle M. White, Nina Duncan, Joy Bergmann, Robert D. Cole, Vinay Parikh, \& Debra A. Bangasser

Department of Psychology and Neuroscience Program, Temple University, Philadelphia, PA 19122

Corresponding author information:

Debra Bangasser, Ph.D.

Temple University

Department of Psychology and Neuroscience Program

1701 North 13th Street

873 Weiss Hall

Philadelphia, PA 19122

Office Phone: 1-215-204-1015

Email: debra.bangasser@temple.edu

Article type: Research Paper

Number of text pages: 26

Number of tables: 1

Number of figures: 4 


\begin{abstract}
Background: Sustained attention, the ability to detect rare and unpredictable events, is central to cognitive performance. This construct can be tested in rodents using a Sustained Attention Task (SAT), where rats are trained to detect an unpredictably occurring signal (a brief light presentation) from non-signal events. The traditional version of this task utilizes an operant chamber with a central panel light for the signal and two retractable response levers. Adaptation of SAT to the increasingly popular touchscreen operant chambers, which do not have levers or fixed lights, could enhance the versatility of the task.
\end{abstract}

New method: Here we developed a touchscreen version of SAT where the light signal is presented in the upper part of the touchscreen, followed by a tone to indicate the beginning of the response period.Rats indicate their choice during this period by touching their nose to one of two touchscreen response areas. The remaining parameters were kept similar to the traditional version

Results: Rats acquired touchscreen SAT at a similar rate to the traditional version. As with the traditional version, shorter stimulus durations on the signaled trials reduced accuracy and the presence of a distractor (a flashing houselight) disrupted performance on the touchscreen version.

Comparison to existing method: Collectively, these data suggest that the touchscreen version is comparable to the traditional version of the SAT, and is an equally valid way of measuring sustained attention.

Conclusions: Many researchers with touchscreen chambers could easily implement our modifications in order to study sustained attention 
Key Words: Attention;Cognition;Operant testing;Touchscreen;Vigilance 


\section{Introduction}

Sustained attention is the ability to detect intermittent and unpredictable events over a prolonged period of time. This elemental component of attention subserves other attentional processes, such as selective and divided attention, and more generally is critical for optimal cognitive performance (Sarter et al., 2001; Smilek et al., 2010). Clinically, disruptions in sustained attention are observed in a range of disorders, including schizophrenia, attention deficit hyperactivity disorder (ADHD), Alzheimer's disease, bipolar disorder, and major depression (Clark et al., 2002; Cornblatt and Keilp, 1994; Gmehlin et al., 2016; Paelecke-Habermann et al.; Perry and Hodges, 1999). Thus, understanding the neurobiological underpinnings of sustained attention, as well as the mechanisms underlying attention dysregulation, are important research goals. One key part of this effort has been the development of laboratory tests of sustained attention for rodents.

A popular operant task to measure sustained attention in rats was developed by McGaughy and Sarter (1995) based on signal detection theory and is referred to, simply, as the Sustained Attention Task (SAT; McGaughy and Sarter, 1995). In this task, rats are trained to discriminate signal from non-signal events presented in a pseudorandom fashion. During a signal trial, a light stimulus of variable duration $(500 \mathrm{~ms}, 50 \mathrm{~ms}$, or $25 \mathrm{~ms})$ is presented from a central panel light, and then the response devices, which are two levers, are extended into the chamber for a short duration of time. The rat is trained to respond to the signal by pressing one of the levers, thereby making a correct response scored as a hit, which is rewarded. To indicate the absence of the light on non-signal trials, the rat responds on the opposite lever, making a correct rejection that is rewarded. Incorrect responses on signal and non-signal trials are considered misses and false alarms, respectively, and are not reinforced. Additional measures 
includeomissions, or trials in which there is no response, and the vigilance index (VI), which is calculated based on the relative number of hits and false alarms excluding omission trials. Finally, to increase attentional demands, a distractor version (dSAT) has been developed during which the overhead houselight flashes, increasing the difficulty of distinguishing signal from non-signal trials (Demeter et al., 2011; Demeter et al., 2008).

SAT is a well-validated way of studying attention and has been utilized to delineate a sustained attention circuit, which includes the basal forebrain corticopetal system (for review see, Demeter and Sarter, 2013; Sarter et al., 2006; Sarter et al., 2001; Sarter et al., 2005). The task has also revealed factors that can contribute to the pathophysiology of certain psychiatric and neurodegenerative disorders (Briand et al., 2008; Kucinski et al., 2013; Parikh et al., 2013). Because of the utility of this task, the SAT procedure has more recently been adapted to test attentional capacities in other species, such as mice and humans, thereby increasing its translational potential (Demeter et al., 2013; Demeter et al., 2008; St Peters et al., 2011). Here we aimed to expand its use even further by altering certain parameters for use in touchscreen operant chambers.

Touchscreen operant chambers are increasing in popularity due to their flexibility. The touchscreen can be easily programmed for different tasks, and cues and levers are not permanently configured for one specific procedure. Other attention tasks, such as the 5-choice serial reaction time task (5-CSRTT) and the continuous performance task (rCPT), which measure sustained and selective attention (Armstrong, 1997; Kremen et al., 1992; Robbins, 2002), have been modified for use in these chambers (Bartko et al., 2011; Kim et al., 2015). In this methods paper, we explain how to adapt SAT to touchscreen operant chambers for rats. The major changes from the traditional version are: 1) the signal light is presented on the touchscreen, 
instead of via a central light panel, 2) rats indicate the presence or absence of the signal by touching with their nose one of two response areas on the touchscreen, instead of pressing an appropriate lever, 3) the response window is indicated with a tone, instead of the presentation of both levers.To validate the touchscreen SAT, we compared acquisitionand performance measures between the traditional (i.e., using an operant chamber with a central panel light and retractable levers) and touchscreen versions.We also assessed performance across different signal durations (500 ms, $50 \mathrm{~ms}$, and $25 \mathrm{~ms}$ ) and in the dSAT manipulation.

\section{Materials and methods}

\subsection{Subjects}

Male Sprague-Dawley rats $(\mathrm{n}=10)$ were obtained from Charles River Laboratories at approximately 60 days of age. The rats were housed individually in standard shoe box cages in a temperature and humidity controlled room with a 12-hour reverse light-dark cycle, with light offset at 8:30am. The rats were given $\sim 7$ days to acclimate before the start of behavioral training.During this time, the rats were handled regularly and given accessto water and standard rat chow ad libitum, allowing free-feeding weights to be established. Three days prior to the start of training rats began food restriction to $85 \%$ of their free-feeding weights. Once training began, there was at least a 30 min delay between session completion and feeding to reduce the formation of an association between task completion and food access.Allexperiments were conducted in accordance with the National Institutes of Health guidelines and were approved by the Temple University Institutional Animal Use and Care Committee.

\subsection{Materials}

Behavioral training and testing took place in four Bussey-Saksida Rat Touch Screen Chambers (Easy-Install System for Rat Touch Screen Systems, Model 80604-20) purchased 
though Lafayette Instrument (Lafayette, IN) and manufactured by (Campden Instruments, Loughbrorough, UK). Each chamber was equipped with a houselight, tone generator, feeder reward area with light (opposite the touchscreen), and camera (Campden Instruments). A sound attenuation cubicle surrounded each chamber. The program was written in the Animal Behavior Environment Test II (ABET II) software and was run via theWhiskerServer controller.This program may be extended to investigators using the Lafayette Touch Screen Chambers upon request. Both $\mathrm{ABET}$ II and the controller software were provided as part of purchase of the touchscreen chambers. One technical note is that Lafayette Instrument sells two feeder reward areas with different sized food troughs. Although rats with cannula implants were not used in this study, in our experience the opening of the large aperture food trough gives rats with cannula implants, electrophysiological, dialysis, or other headstages, much better clearance to retrieve the pellet than the smaller aperture food trough.

To limit response regions on the touchscreen, a thin plastic maskwas inserted directly in front of the touchscreen. The mask contained three openings: one central circular opening through which the signal was presented and two lower square openings that served as response areas. See Figure 1 for a depiction of the dimensions. For reference, with regard to height, a rat's nose will align with the center of the square response areas when it is standing on all four legs. During piloting, we had masks built by a vendor (Everything Plastic, Philadelphia, PA) from two sets of material: black acrylic (3.175 mm thick) and black polycarbonate with one matte finish side (3.175 $\mathrm{mm}$ thick). The rats chewed through the acrylic fairly easily, so we switched to the polycarbonate, which was more resistant to gnawing. The black polycarbonate was positioned so that the matte side faced into the chamber. 
The reward pellets used were $45 \mathrm{mg}$ Bioserve dustless precision pellets, grain-based (product \#F0165). Prior to being loaded into the food dispensers, pellets were sifted with a flour sifter to remove additional dust that can clog the food dispensers. Chambers were cleaned with $10 \%$ alcohol after each use.

\subsection{TouchscreenSAT Training}

\subsubsection{Initial shaping phase}

Training took place 6 days per week from $10 \mathrm{am}-3 \mathrm{pm}$ and rats were run in the same order and same chamber each day. Each day, prior to running a behavior schedule, the boxes were tested to ensure that touchscreens were displaying the correct stimuli and the food dispenser was working properly.

The first shaping phase was Pokeshape. During this schedule, rats were trained to make nose-pokes under a fixed-ratio one (FR1) schedule of reinforcement. Nose-pokes in either of the two response areaswere rewarded, however, pokes were not reinforced in an area when the side differential was five or greater in favor of that side. This was done to prevent the development of a side bias towards either the left or right response areas. During this phase there were no signals displayed and the houselight was off. For all schedules including Pokeshape, thefeeder reward area was illuminated to indicate the presence of a pellet and then the light was turned off when the pellet was retrieved. A Pokeshape session lasted 40 min or until 120 pokes were rewarded. Passing criteria for this phase was defined as two consecutive days of 120 nose-pokes in the allotted time.

\subsubsection{Overview of schedules}

After the rats were shaped to poke for food, they were run through three shaping schedules (Training Phases 1-3) before they were moved to the SAT schedule (Table 1). There 
are some commonalities between all of the training and SAT schedules that are worth noting before the differences are detailed. All of the schedules consisted of 162 trials that were divided into three blocks of 54 trials each. Signal and non-signal trials were presented in a pseudorandom fashion such that the same number of signal and non-signal trials occurred in each of the three blocks.

During each trial, a signal or non-signal event was presented, followed by a $4 \mathrm{~s}$ response window during which the rats could make a response. An omission was counted when no response was made during the response window. One modification for the touchscreen version was the indication of the response window. In the traditional method, events (i.e., signals or nonsignals) occur while the levers are retracted and thento indicate the beginning of the response window the levers are presented. Because the touchscreen chambers lack retractable levers, the start of the response window was indicated with a $200 \mathrm{~ms}, 70 \mathrm{~dB}$ tone $(3 \mathrm{kHz})$ beginning $1 \mathrm{~s}$ after a signal or non-signal event. This is similar to Gill et al. (2000), where levers were extended into the chamber throughout the session and the response window was indicated with a tone.

The initiation of each trial was the same for the training and SAT schedules. At the start of the session, a food pellet was automatically delivered, and the first intertrial interval (ITI) began when an infrared beam break indicated that the rat had removed its head from the food reward area. Similarly, on correct trials, the ITI began after the rat removed its head from the reward area. On incorrect and omission trials, the ITI began following the response or the end of the response window, respectively. For all trials, the ITI was set at $9 \pm 3 \mathrm{~s}$. The session was terminated after 162 trials were completed which took approximately 60 min for Training Phase 1, $45 \mathrm{~min}$ for the other training phases, and $40 \mathrm{~min}$ for SAT.

\subsubsection{Training schedules}


Training Schedule 1 was when rats were initially trained to discriminate between signal and non-signal trials.To facilitate the acquisition of this task, the houselight remained off for easier detection of the light stimulus during signal trials andall signals were $500 \mathrm{~ms}$ in duration. While correct responses were rewarded with a food pellet, incorrect responses triggered a series of correction trials in which the previous trial type (i.e., signal or non-signal) was presented again. The series of correction trials ended when a correct response was made or when the rat made four consecutive incorrect responses. Note that correction trials did not count towards the 162 total trials for Training Phase 1. Training Phase 2 was similar to Training Phase 1, except there were no correction trials. The only change from Training Phase 2 to Training Phase 3 was that the houselight was turned on, which made the signal more difficult to detect, and thus increased the attentional demands of the task. Criteria performance on each of these training schedules was defined as at least $70 \%$ hits, at least $70 \%$ correct rejections, and less than $20 \%$ omissions for three consecutive days.

\subsubsection{SAT schedule}

After reaching criteria on Training Phase 3, rats advanced to the SAT schedule. Unlike the training phases where the signal was always $500 \mathrm{~ms}$, during SAT there were three signal durations: $500 \mathrm{~ms}, 50 \mathrm{~ms}$, and $25 \mathrm{~ms}$. These signals were presented in a pseudorandom fashionso that there was an equal number of each signal duration per trial block. The shorter signal durations increase attentional demands, allowing for within session comparisons of stimuli that tax the attention system to varying degrees. Criteria performance on SAT was defined as at least $70 \%$ hits at the $500 \mathrm{~ms}$ stimulus duration, at least $70 \%$ correct rejections, and less than $20 \%$ omissions for three consecutive days. 
Note that occasionally during training or SAT, rats developed a bias where they began disproportionately responding to one side of the touchscreen, leading to a large difference in correct responses for the two types of events (e.g., $80 \%$ correct on non-signal trials, $30 \%$ on signal trials). When this occurred, rats were returned back to Training Phase 1, because the correction trials encouraged correct responses to both events. Rats were kept on Training Phase 1 for 5 days or until passing.

\subsection{5dSAT}

After rats acquired SAT, a subsetof animals was given one session of the dSAT. In dSAT the houselight flashedat a frequency of $0.5 \mathrm{~Hz}$ only during the second of the three blocks of trials. Thus, performance on SAT in the blocks before and after the distractor could be assessed within session.

\subsection{Traditional SAT Training}

Data regarding acquisition time and performance measures on a traditional version of SAT were included from a previous study in which these measures were not reported (Cole et al., 2016). As in the current study, adult male Sprague-Dawley rats $(n=8)$ were trained on the task and used to collect acquisition rate data, but one animal was dropped from baseline performance analyses due to a health issue. Further details on the traditional SAT procedure have been described previously (Cole et al., 2016; Parikh et al., 2013), so here we will just highlight the major differences between the two procedures.

As noted above, the major differences between the traditional and touchscreen SAT versions are: the signal light device, response devices, and the way the onset of the response window is indicated. Additional differences involved the type of reward and location of its administration. The data presented here on the traditional SAT version were collected in 
chambers that provided water rewards through a liquid receptacle located directly below the central light panel (i.e., on the same side as the signal and response devices). As noted, in the touchscreen version, rewards were provided through a food receptacle located opposite the touchscreen. Importantly, however, a similar configuration and food rewards were used in the original McGaughy and Sarter (1995) 2-lever version of the task. In addition to this difference in the equipment, there was also a difference in training procedure between the two SAT versions. In the traditional SAT version used here, the houselight was left on during the shaping and all training phases. However, in the touchscreen version it was only turned on during Training Phase 3. Again, however, this is similar to the original McGaughy and Sarter (1995) operant version of the task.

\subsection{Performance measures}

The performance measures to assess sustained attention in our touchscreen procedure were based on an operant SAT procedure originally developed by McGaughy and Sarter (1995). The percentage of hits was calculated as a measure of correct responses on signal trials. To quantify correct responses on non-signal trials, the percentage of correct rejections was calculated. The percentage of omissions was also assessed. Finally, we calculated the vigilance index (VI), which is based on the proportion of hits (h) and false alarms (f), using the following

formula: $V I=\frac{h-f}{2 \times(h+f)-(h+f)^{2}}$. Note that VI does not include omitted trials as recommended by Frey and Collier (1973). The values of VI range from -1 to +1 with a value of 0 indicating that the rat cannot distinguish between signal and non-signal trials and positive values indicating increasing accuracy.

\subsection{Statistics}


A Mann-Whitney U test was used to analyze the number of days to reach criteria on SAT in the traditional vs. touchscreen version. After rats attained criteria on SAT, performance measures from one day of SAT testing were compared between the traditional and touchscreen versions using independent samples t-tests (2-tailed), unless Levene's test for equality of variances was significant, in which case Welch's t-test was used instead. Repeated measures ANOVAs were used to compare performance at the different signal durations for SAT and to assess the effect of the distractor in dSAT. Bonferroni corrected post-hoc tests were used to compare differences.

\section{Results}

\subsection{Description of touchscreen SAT performance}

A qualitative assessment of performance on touchscreen operant SAT revealed that rats were able to learn and perform this version of the task. The provided video shows an example of one subject's baseline performance on the touchscreen SAT schedule. Unlike the traditional version where the levers are only available when the response is required, in the touchscreen version the response areas are present throughout all phases of the task. Thus in the touchscreen version, rats often adopt a strategy of frequently poking the response areas, particularly the non-signal response area, throughout the ITI while watching for the signal. This strategy allows them to make the most rapid response possible during a nonsignal trial, while also being close to the touchscreen to move to the response area for the signal on a signaled trial. This additional poking does not seem to impair performance, as detailed below. Additionally, we have observed that some rats on the traditional version try to engage with the retracted lever ports during the ITI, suggesting that if they had the opportunity to lever press they would engage in lever pressing. 


\subsection{Comparisons between performance on traditional and touchscreen SAT}

Several measures of performance were compared between the traditional and the touchscreen SAT task. First, the number of trials to reach criteria on SAT was analyzed for each version and no significant difference was found between the traditional and touchscreen SAT versions ( $U=37, Z=0.26, p=.829)$ (Fig. 2a). However, one subject in the touchscreen group took substantially longer to reach training criteria, and a second subject did not reach criteria, perhaps suggesting more variability in training time for the touchscreen SAT procedure than the traditional version.

After rats on both SAT versions reached criteria, their performance on one session was compared. Rats on the traditional and touchscreen SAT versions had a similar percentage of hits $[t(14)=0.040, p=.969]$ and correct rejections $[t(9.527)=.629, p=.544]$, suggesting that the accuracy on both signal and non-signal trials was not different when using the touchscreens (Fig. $2 \mathrm{~b}, \mathrm{c})$. VI scores were also comparable between the two versions $[t(14)=.673, p=.512]$ (Fig. $2 \mathrm{~d}$ ). Omissions were low on both versions and there were no significant differences on this measure $[t(12.897)=-1.693, p=.115]$ (Fig. 2e). Overall, these data suggest that the modifications made for the touchscreen SAT version did not significantly affect performance.

\subsection{Effects of signal length and the distractor condition}

Performance was compared between the different signal durations on the touchscreen version of SAT. There was a main effect of stimulus duration $[F(2,16)=25.06, p<.001]$, with rats performing better at the longest stimulus duration of $500 \mathrm{~ms}$, than at $50 \mathrm{~ms}(p<.001)$ or 25 ms $(p=.002)$. There was no significant difference in performance between 50 msand 25 msstimulus duration $(p=.127)$ (Fig. 3a). 
Rats run using the touchscreen boxes were also tested in dSAT, during which the houselight flashed in order to increase attentional demands during the second block of trials. Attentional performance was affected by presentation of the visual distractors (main effect of block; $[F(2,12)=15.64, p<.001])($ Fig. $3 b)$. Post-hoc tests revealed that performance on Block 2was worse than performance on Blocks $1(p=.017)$ and $3(p=.003)$.

\section{Discussion}

The goal of this study was to develop a version of SAT that could be run on touchscreen operant chambers. To accomplish this, the traditional SAT version was modified such that the signal was presented in the center of the touchscreen and responses were made on two areas of the touchscreen located below and on either side of the signal area.Additionally, the beginning of the response window (i.e., the period after each event when a nose poke was recorded as a response) was indicated with a tone.These modifications did not significantly alter this task because performance on touchscreen SAT was comparable to performance on traditional SAT. Additionally, performance on touchscreen SAT declined with shorter signal durations and also was impaired by the distractor (i.e., flashing houselight), suggesting that, similar to the traditional SAT version,touchscreen SAT can be manipulated to increase attentional demands.

\subsection{Comparison of touchscreen SAT versus traditional SAT}

As noted, performance on touchscreen SAT and traditional SAT were directly compared. Specifically, acquisition in both versions was assessed by quantifying the number of days to reach criteria. There was no statistical difference between traditional SAT and touchscreen SAT in rates of acquisition.

Once criteria were met, performance on both signal and non-signal trials was found to be comparable between rats trained on the traditional and touchscreen versions of SAT. The 
vigilance index was also similar in both versions. The result for non-signal trials is notable because other researchers have suggested that the use of cues, such as tones, to indicate the beginning of the response window increases false alarms (St. Peters et al., 2011). However, we did not find a significant difference in the number of correct rejections (i.e., the inverse of false alarms) between the traditional and touchscreen versions of SAT. Thus, in our hands at least, the use of the tone did not increase false alarms.

Omissions were very low on both SAT versions, although there was a small but not significant increase in omissions in the touchscreen SAT version compared to traditional SAT. This increase could be due to the use of the tone to indicate the response window, as it may be less salient than levers extending into the boxes. However, the similar rates of acquisition and performance on traditional and touchscreen versions of SAT, along with the low number of omissions on the touchscreen version, suggest that the use of the tone does not adversely affect performance. An alternative explanation for the slight but not statistically significant increase in omissions on touchscreen SAT could be the use of food reward, which would be consistent with previous publications revealing more omissions with a food compared to water reward (McGaughy and Sarter, 1995; Parikh et al., 2013). Yet, the very low number of omissionson touchscreen SAT suggests that rats are highly motivated to perform the task, even for the food reward.

\subsection{Increasing attentional demands}

A useful feature of an attention task is the ability to assess performance under conditions that increase attentional demands. One way this is accomplished in the traditional SAT procedure, is through the use of three different signal durations (500 ms, $50 \mathrm{~ms}$, and $25 \mathrm{~ms}$ ) on signal trials, with the shorter stimulus durations requiring greater vigilance to be reliably detected 
(McGaughy and Sarter, 1995). The touchscreen SAT version we developed uses the same three signal durations and, similar to traditional SAT, performance in the touchscreen version was found to worsen when the signal durations were shorter. In the touchscreen version performance declined between the $50 \mathrm{~ms}$ and $25 \mathrm{~ms}$ signal duration, however, performance for these two stimulus durations was not significantly different. To achieve a more graded level of performance on signal trials, the middle signal duration could be increased. In fact, Newman and McGaughy (2008) used a $100 \mathrm{~ms}$, rather than $50 \mathrm{~ms}$, middle signal duration and they found a greater distinction in performance between $100 \mathrm{~ms}$ and $25 \mathrm{~ms}$ than we did between $50 \mathrm{~ms}$ and 25 ms. Future studies could confirm this using the touchscreen boxes.

Another way to increase attentional demands is to use the dSAT procedure. Mimicking the findings from the traditional version (Howe et al., 2010), in touchscreen dSAT, performance decreased in the presence of the distractor during the second block of trials, but recovered when the distractor ceased (i.e., during the third trial block). Thus, touchscreen dSAT successfully captures the effects of increased attentional demands. Collectively, the similarities in performance on the traditional and touchscreen SAT versions, particularly under conditions that increased attentional demands, suggest the touchscreen SAT version is a valid measure of sustained attention.

\subsection{Using touchscreen SAT for other strains and species}

The traditional SAT version has been used with a variety of rat strains, such as Fischer/Brown Norway hybrid rats, Wistar rats, and Long-Evans rats (McGaughy and Sarter, 1995; Newman and McGaughy, 2008; Parikh et al., 2013). We chose Sprague-Dawley rats for these studies because of our previous work with this species (e.g., Cole et al., 2016). Touchscreen SAT acquisition and performance in Sprague-Dawley rats is expected to be 
comparable with other rat strains. In fact, it is possible that pigmented rat strainswould actually acquire SAT faster than the albino Sprague-Dawley strain used here because pigmented strains have greater visual acuity(Prusky et al., 2002). Arguing against this, however, is data revealing that Sprague-Dawley and pigmented Lister Hooded rats have similar acquisition rates on a touchscreen visual discrimination task, where rats must distinguish between two black and white images(Bussey et al., 2008). Whether or not differences in acquisition are found between rat strains, it is expected that, just as with the traditional version, a variety of rat strains could reach stable performance on touchscreen SAT.

The ability to easily manipulate genes in mice has increased the demand for cognitive tests in this species. To meet this demand, St. Peters et al. (2011) developed a mouse version of the SAT that takes place in a traditional operant chamber, but instead of retractable levers, retractable nose-poke devices are used. Given that mice can learn this version of SAT, it is likely that they could also learn a touchscreen version. In fact, touchscreen versions of 5-CSRTT task and $\mathrm{rCPT}$ have been developed for mice, indicating that mice can acquire touchscreen attention tasks (Kim et al., 2015; Romberg et al., 2011).

\subsection{Conclusions}

Sustained attention is critical for optimal cognition (Sarter et al., 2001). Additionally, attentional disruptions are a shared feature of many psychiatric disorders(Clark et al., 2002; Cornblatt and Keilp, 1994; Gmehlin et al., 2016; Paelecke-Habermann et al.; Perry and Hodges, 1999). Therefore, understanding the mechanisms that impair and promote sustained attention will be critical for developing treatments to improve cognition, especially in patients suffering from attentional impairments. Yet, relative to other cognitive processes (e.g., spatial memory, motor learning, etc.), sustained attention has been understudied by basic neuroscience 
researchers. This relative neglect is probably due, in part, to the specialized equipment required. The development of the touchscreen operant SAT procedure increases the usefulness of this procedure as it can easily be run in the increasingly popular and versatile touchscreen chambers. We hope that this advance will broaden access to this method for researches interested in understanding the neurobiology of cognition. 


\section{Acknowledgements}

We would like to thank Sarah Cohen, Madeleine Salvatore, and Attilio Cerettifor their assistance with the behavior, and Brittney Yegla for her technical assistance. This work was supported by the National Institutes of Healthgrant NIMH 092438 and National Science Foundation grant IOS 1552416 to D.A.B. 


\section{References}

Armstrong C. Selective versus sustained attention: A continuous performance test revisited. The Clinical Neuropsychologist, 1997; 11: 18-33.

Bartko SJ, Romberg C, White B, Wess J, Bussey TJ, Saksida LM. Intact attentional processing but abnormal responding in M1 muscarinic receptor-deficient mice using an automated touchscreen method. Neuropharmacology, 2011; 61: 1366-78.

Briand LA, Flagel SB, Garcia-Fuster MJ, Watson SJ, Akil H, Sarter M, Robinson TE. Persistent alterations in cognitive function and prefrontal dopamine D2 receptors following extended, but not limited, access to self-administered cocaine.

Neuropsychopharmacology, 2008; 33: 2969-80.

Bussey TJ, Padain TL, Skillings EA, Winters BD, Morton AJ, Saksida LM. The touchscreen cognitive testing method for rodents: how to get the best out of your rat. Learn Mem, 2008; 15: 516-23.

Clark L, Iversen SD, Goodwin GM. Sustained attention deficit in bipolar disorder. Br J Psychiatry, 2002; 180: 313-9.

Cole RD, Kawasumi Y, Parikh V, Bangasser DA. Corticotropin releasing factor impairs sustained attention in male and female rats. Behav Brain Res, 2016; 296: 30-4.

Cornblatt BA, Keilp JG. Impaired attention, genetics, and the pathophysiology of schizophrenia. Schizophr Bull, 1994; 20: 31-46.

Demeter E, Guthrie SK, Taylor SF, Sarter M, Lustig C. Increased distractor vulnerability but preserved vigilance in patients with schizophrenia: evidence from a translational Sustained Attention Task. Schizophrenia research, 2013; 144: 136-41.

Demeter E, Hernandez-Garcia L, Sarter M, Lustig C. Challenges to attention: a continuous arterial spin labeling (ASL) study of the effects of distraction on sustained attention. Neuroimage, 2011; 54: 1518-29.

Demeter E, Sarter M. Leveraging the cortical cholinergic system to enhance attention. Neuropharmacology, 2013; 64: 294-304.

Demeter E, Sarter M, Lustig C. Rats and humans paying attention: cross-species task development for translational research. Neuropsychology, 2008; 22: 787-99.

Frey PW, Colliver JA. Sensitivity and responsivity measures for discrimination learning. Learning and Motivation, 1973; 4: 327-42.

Gill TM, Sarter M, Givens B. Sustained visual attention performance-associated prefrontal neuronal activity: evidence for cholinergic modulation. J Neurosci, 2000; 20: 4745-57.

Gmehlin D, Fuermaier AB, Walther S, Tucha L, Koerts J, Lange KW, Tucha O, Weisbrod M, Aschenbrenner S. Attentional Lapses of Adults with Attention Deficit Hyperactivity Disorder in Tasks of Sustained Attention. Archives of clinical neuropsychology : the official journal of the National Academy of Neuropsychologists, 2016; 31: 343-57.

Howe WM, Ji J, Parikh V, Williams S, Mocaer E, Trocme-Thibierge C, Sarter M. Enhancement of Attentional Performance by Selective Stimulation of [alpha]4[beta]2[ast] nAChRs: Underlying Cholinergic Mechanisms. Neuropsychopharmacology, 2010; 35: 1391-401.

Kim CH, Hvoslef-Eide M, Nilsson SR, Johnson MR, Herbert BR, Robbins TW, Saksida LM, Bussey TJ, Mar AC. The continuous performance test (rCPT) for mice: a novel operant touchscreen test of attentional function. Psychopharmacology (Berl), 2015; 232: 3947-66. 
Kremen WS, Seidman LJ, Faraone SV, Pepple JR, Tsuang MT. Attention/informationprocessing factors in psychotic disorders. Replication and extension of recent neuropsychological findings. J Nerv Ment Dis, 1992; 180: 89-93.

Kucinski A, Paolone G, Bradshaw M, Albin RL, Sarter M. Modeling fall propensity in Parkinson's disease: deficits in the attentional control of complex movements in rats with cortical-cholinergic and striatal-dopaminergic deafferentation. J Neurosci, 2013; 33: 16522-39.

McGaughy J, Sarter M. Behavioral vigilance in rats: task validation and effects of age, amphetamine, and benzodiazepine receptor ligands. Psychopharmacology (Berl), 1995; 117: 340-57.

Newman LA, McGaughy J. Cholinergic deafferentation of prefrontal cortex increases sensitivity to cross-modal distractors during a sustained attention task. J Neurosci, 2008; 28: 264250 .

Paelecke-Habermann Y, Pohl J, Leplow B. Attention and executive functions in remitted major depression patients. J Affect Disorders; 89: 125-35.

Parikh V, Howe WM, Welchko RM, Naughton SX, D'Amore DE, Han DH, Deo M, Turner DL, Sarter M. Diminished trkA receptor signaling reveals cholinergic-attentional vulnerability of aging. Eur J Neurosci, 2013; 37: 278-93.

Perry RJ, Hodges JR. Attention and executive deficits in Alzheimer's disease. A critical review. Brain : a journal of neurology, 1999; 122 ( Pt 3): 383-404.

Prusky GT, Harker KT, Douglas RM, Whishaw IQ. Variation in visual acuity within pigmented, and between pigmented and albino rat strains. Behav Brain Res, 2002; 136: 339-48.

Robbins TW. The 5-choice serial reaction time task: behavioural pharmacology and functional neurochemistry. Psychopharmacology (Berl), 2002; 163: 362-80.

Romberg C, Mattson MP, Mughal MR, Bussey TJ, Saksida LM. Impaired Attention in the 3xTgAD Mouse Model of Alzheimer's Disease: Rescue by Donepezil (Aricept). The Journal of Neuroscience, 2011; 31: 3500-7.

Sarter M, Gehring WJ, Kozak R. More attention must be paid: the neurobiology of attentional effort. Brain Res Rev, 2006; 51: 145-60.

Sarter M, Givens B, Bruno JP. The cognitive neuroscience of sustained attention: where topdown meets bottom-up. Brain Res Brain Res Rev, 2001; 35: 146-60.

Sarter M, Hasselmo ME, Bruno JP, Givens B. Unraveling the attentional functions of cortical cholinergic inputs: interactions between signal-driven and cognitive modulation of signal detection. Brain Res Brain Res Rev, 2005; 48: 98-111.

Smilek D, Carriere JSA, Cheyne JA. Failures of sustained attention in life, lab, and brain: Ecological validity of the SART. Neuropsychologia, 2010; 48: 2564-70.

St Peters M, Cherian AK, Bradshaw M, Sarter M. Sustained attention in mice: expanding the translational utility of the SAT by incorporating the Michigan Controlled Access Response Port (MICARP). Behav Brain Res, 2011; 225: 574-83.

St. Peters M, Cherian AK, Bradshaw M, Sarter M. Sustained attention in mice: Expanding the translational utility of the SAT by incorporating the Michigan Controlled Access Response Port (MICARP). Behavioural Brain Research, 2011; 225: 574-83. 


\section{Figure Captions}

Table 1. Depiction of the training schedules.

Figure 1. Depiction of the dimensions of the mask placed in front of the touchscreen.

Figure 2. Comparisonsof performance between rats trained on the traditionaland touchscreenversions of the SAT. A) The number of days to reach criteria was similar between traditional $(\mathrm{n}=8)$ and touchscreen $(\mathrm{n}=10)$ SAT. B) Percent hits and C) percent correct rejections were comparable for both the traditional $(n=7)$ and touchscreen versions $(n=9)$ of SAT. D) The vigilance index, an overall measure of performance, was also similar for both versions. E) The percentage of trials omitted was low for both versions and there was no significant difference between them. Panels B-E present means \pm SEM.

Figure 3. Increasing the attentional demands impairs attentionin rats $(n=9)$ trained on the touchscreen version of SAT. A) Vigilance index decreases as the signal duration shortens. Asterisks indicate a significant difference $(p<.05)$ from the $500 \mathrm{~ms}$ signal duration. B) Rats run on touchscreen dSAT show decreased vigilance during Block 2 (indicated with a dark bar), when the houselight flashes rapidly to increase attentional demand.Asterisk indicates a significant different from Block 1 and Block $3(p<.05)$. Both panels present means \pm SEM. 


\begin{tabular}{|c|c|c|c|c|}
\hline $\begin{array}{c}\text { Training } \\
\text { Stage }\end{array}$ & $\begin{array}{c}\text { Max. } \\
\text { Trials }\end{array}$ & $\begin{array}{c}\text { Signal } \\
\text { Duration }(\mathbf{m s})\end{array}$ & $\begin{array}{c}\text { Special } \\
\text { Conditions }\end{array}$ & Criteria \\
\hline Pokeshape & 120 & No events & Houselight off & 120 trials in 40 minutes \\
\hline $\begin{array}{c}\text { Training } \\
\text { Phase 1 }\end{array}$ & 162 & 500 & $\begin{array}{c}\text { Houselight off, } \\
\text { Correction trials }\end{array}$ & $\begin{array}{c}>70 \% \text { Hits, }>70 \% \text { CR, } \\
<20 \% \text { Omissions for } \\
3 \text { consecutive days }\end{array}$ \\
\hline $\begin{array}{c}\text { Training } \\
\text { Phase 2 }\end{array}$ & 162 & 500 & Houselight off & $\begin{array}{r}>70 \% \text { Hits, }>70 \% \text { CR, } \\
<20 \% \text { Omissions for } \\
3 \text { consecutive days }\end{array}$ \\
\hline $\begin{array}{c}\text { Training } \\
\text { Phase 3 }\end{array}$ & 162 & 500 & Houselight on & $\begin{array}{r}>70 \% \text { Hits, > 70\% CR, } \\
<20 \% \text { Omissions for } \\
3 \text { consecutive days }\end{array}$ \\
\hline SAT & 162 & $500,50,25$ & Houselight on & $\begin{array}{r}>70 \% \text { Hits, }>70 \% \text { CR, } \\
<20 \% \text { Omissions for } \\
3 \text { consecutive days }\end{array}$ \\
\hline
\end{tabular}




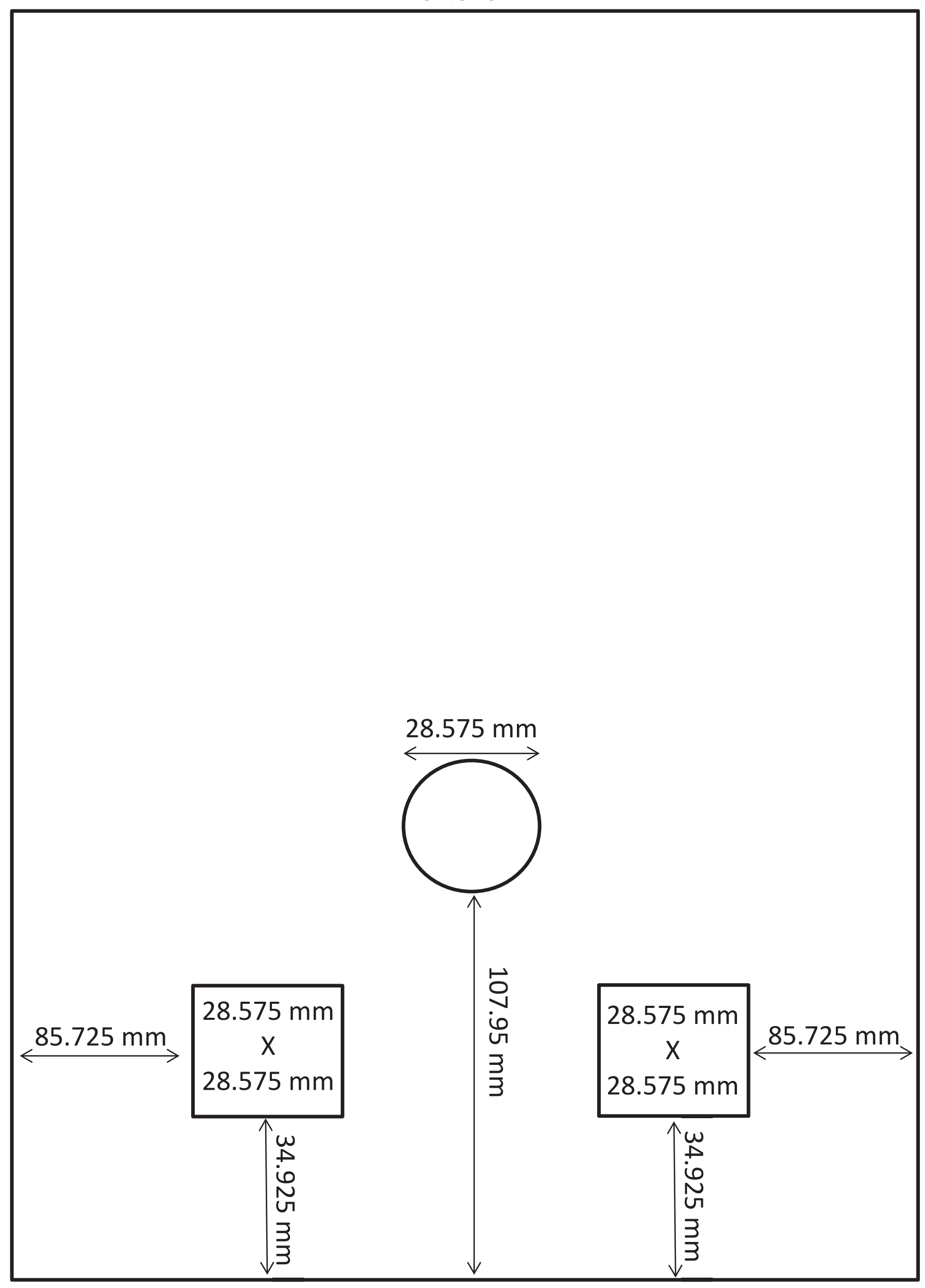

$\omega$
$\sigma$
$\sigma^{2}$
N
3
3 
A) Trials to Criteria

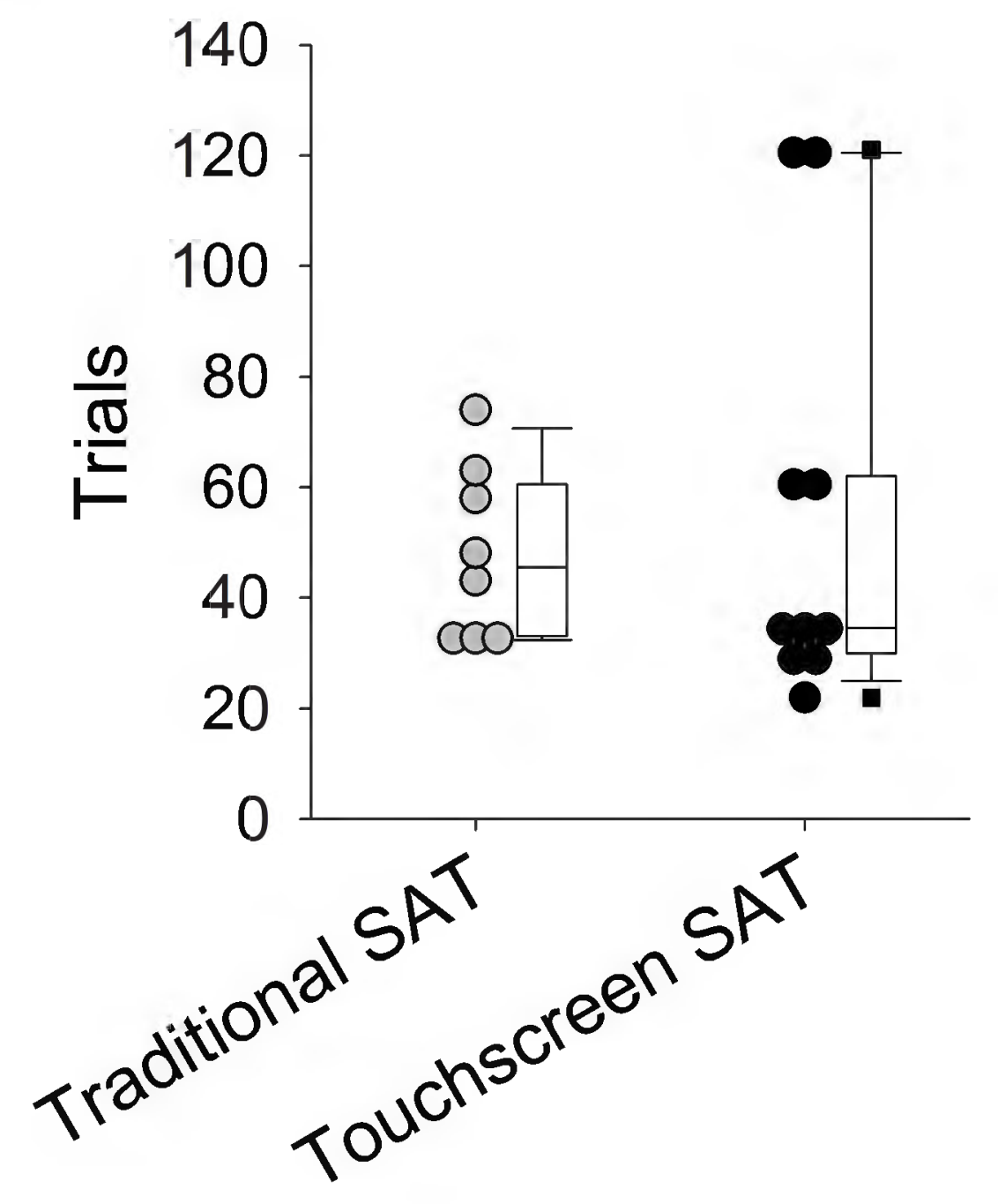

Hits

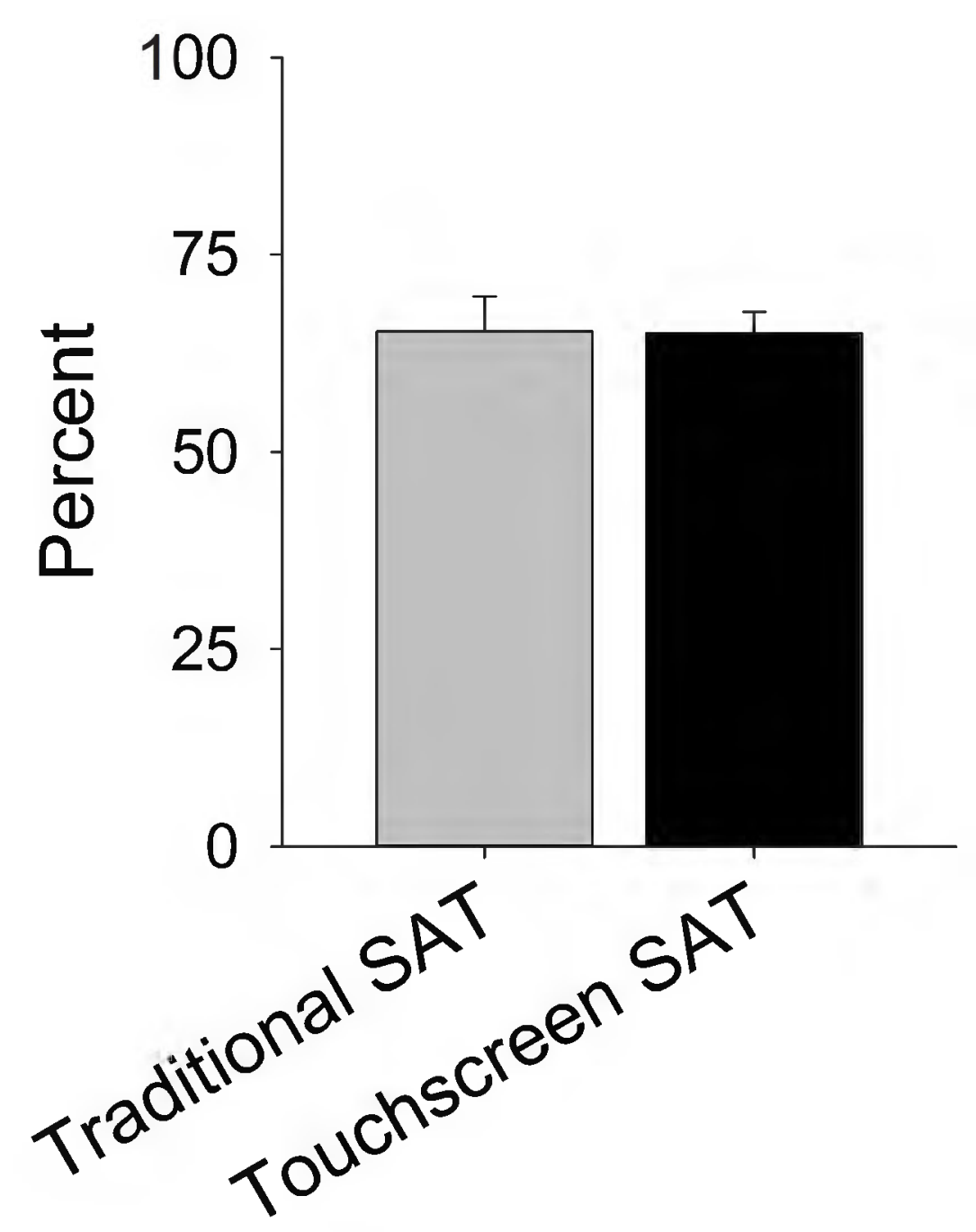

D) Vigilance

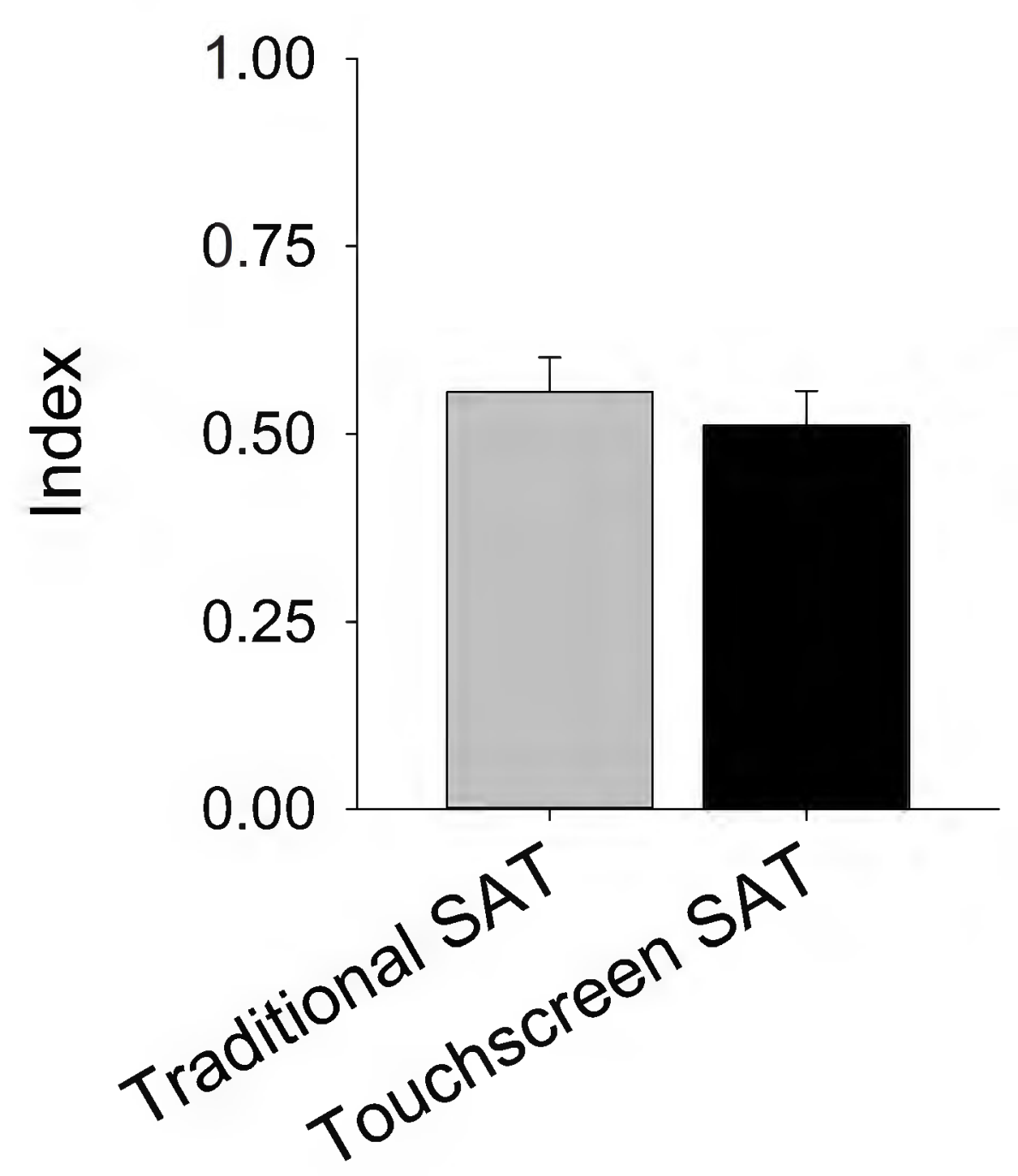

C) Correct Rejections

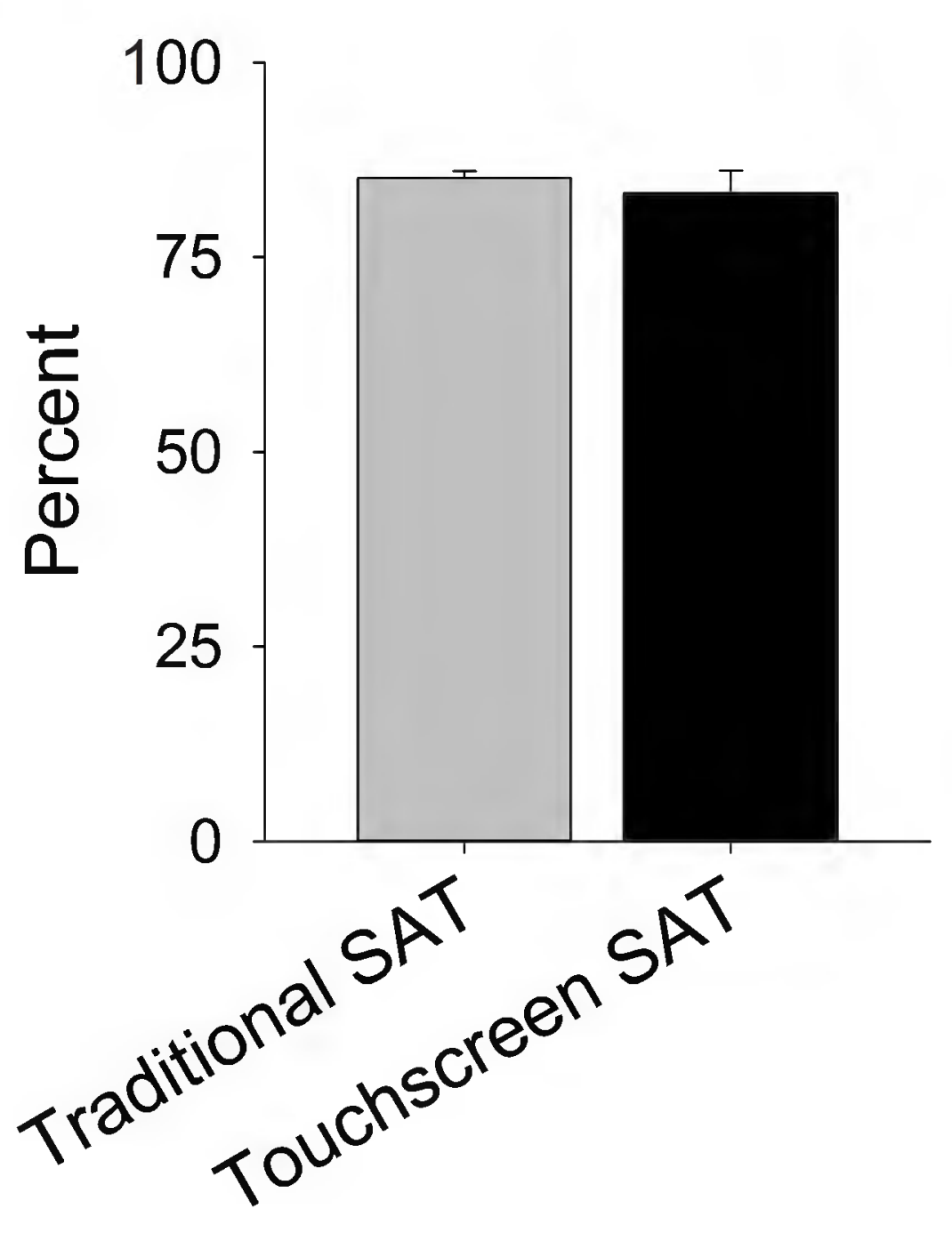

E) Omissions

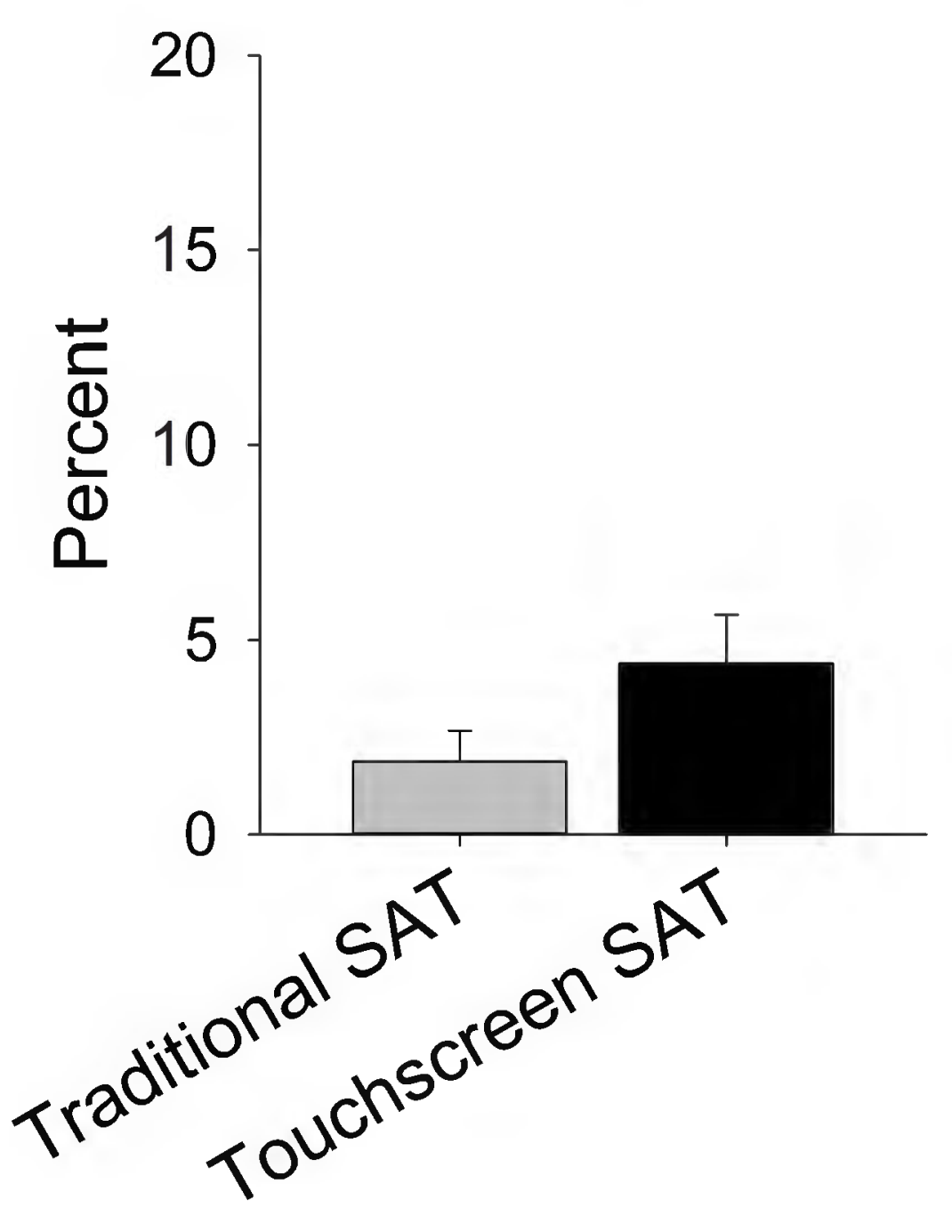




\section{Signal Durations}

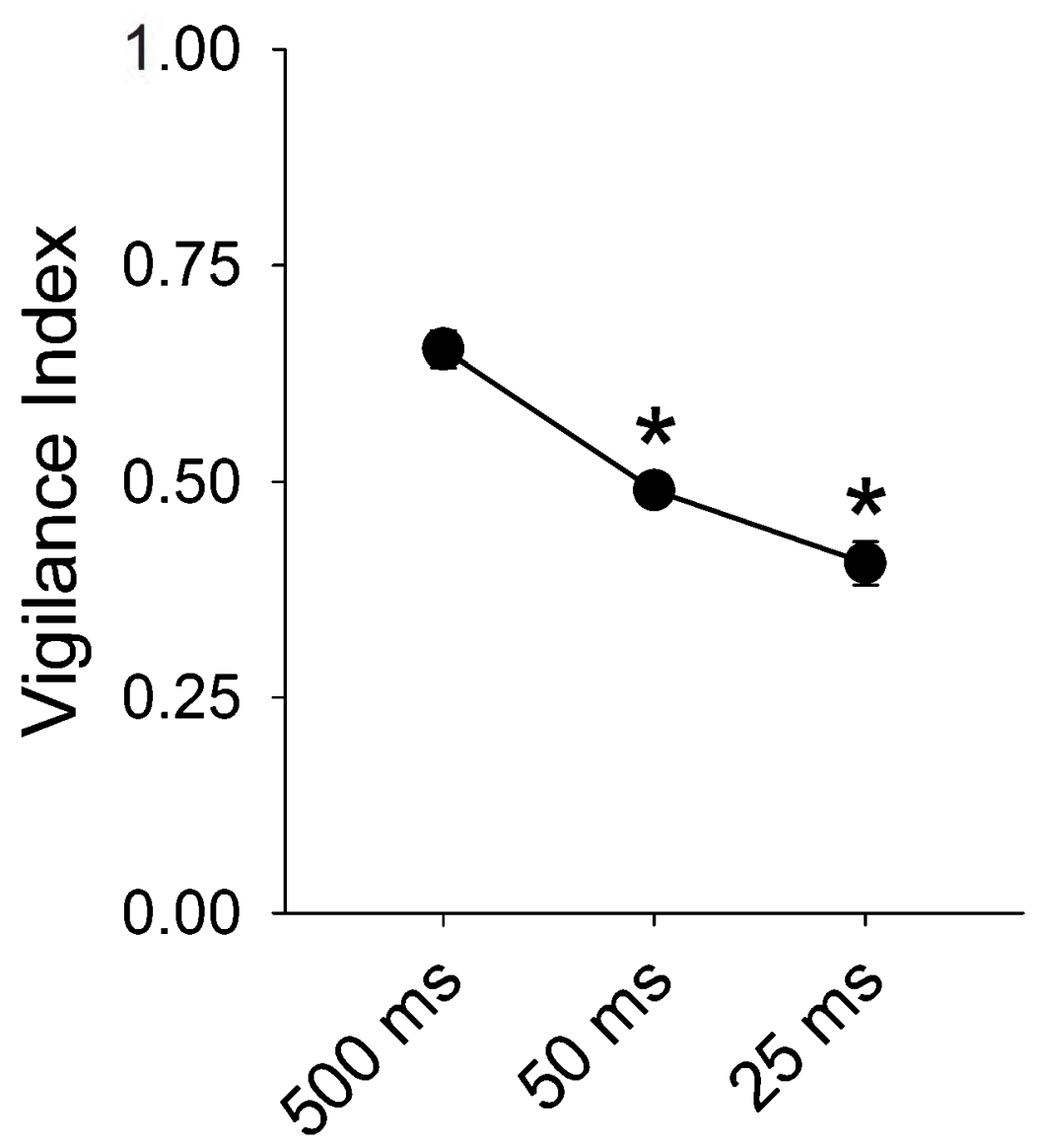

dSAT

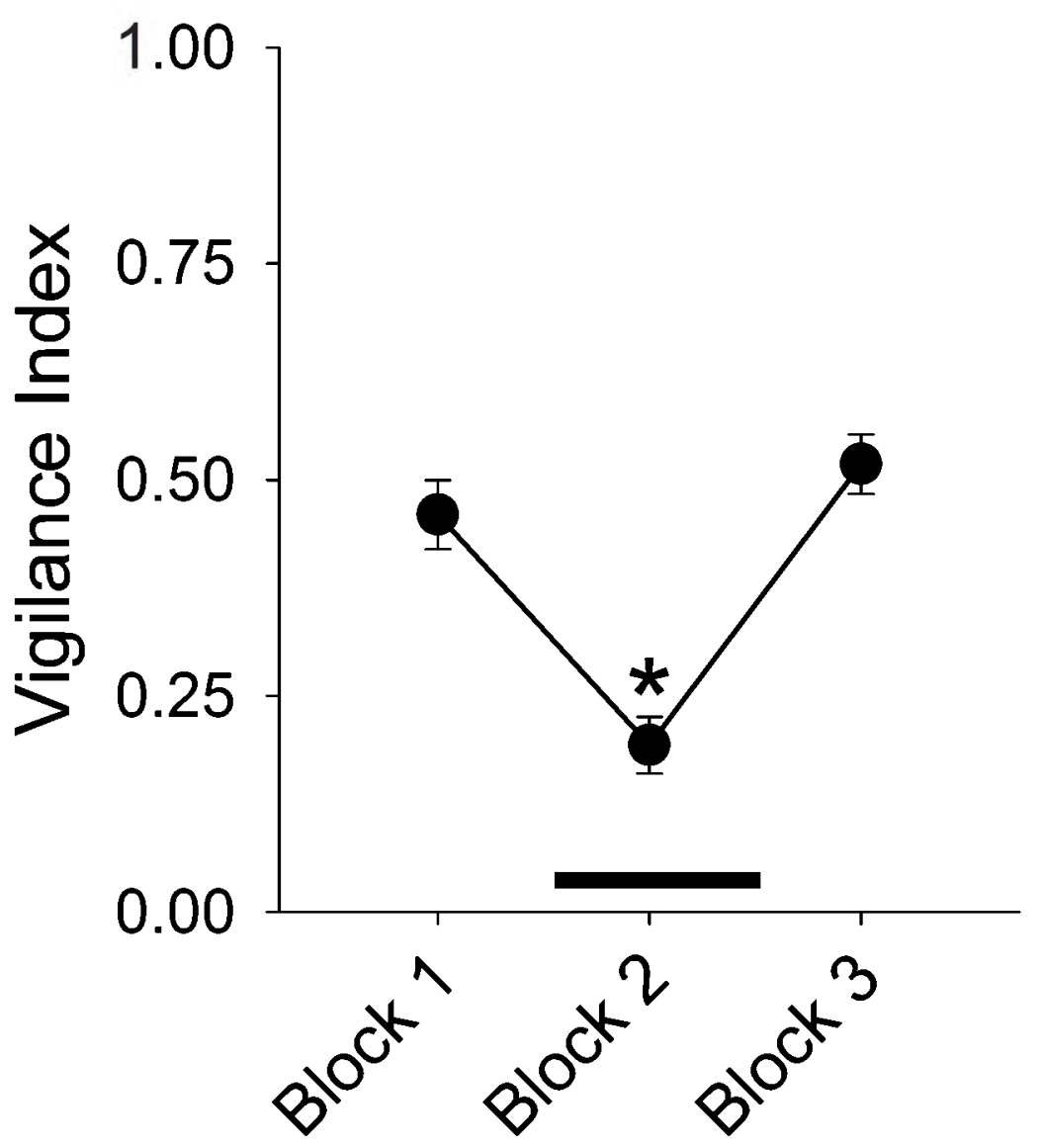

